\title{
Evaluación de diferentes tratamientos para reducir el efecto de Dothistroma septospora en Pinus radiata de tres años*
}

\author{
Evaluation of different treatments to reduce the impact of \\ Dothistroma septospora on three-year old Pinus radiata \\ C.D.O.: $414: 412$
}

RODOLFO H. CONTRERAS

Forestal Alihuén, Letelier 236 Of. 302, Valdivia, Chile.

\section{RESUMEN}

El presente trabajo pretende hacer claridad sobre la estimación de la pérdida de crecimiento que provoca el daño causado por Dothistroma septospora al Pino Insigne y sobre la recuperación de la misma que se obtiene con diversos tratamientos. Se definen seis tratamientos más un testigo, que incluyen la aplicación de fertilizante, herbicida y fungicida separadamente y uno que combina los dos últimos, todos aplicados al establecimiento de una plantación de Pinus radiata. Asimismo se incluyen dos umbrales biológicos de crecimiento con aplicación anual de fungicida con y sin herbicida al establecimiento.

El diámetro mostró ser más adecuado que la altura para medir la eficiencia de los tratamientos. La aplicación del fungicida al primer año reduce significativamente la intensidad del daño, pero no supera al umbral de crecimiento. La aplicación de herbicida al establecimiento y fungicida, un año después, supera en $20 \%$ el crecimiento del testigo.

\section{SUMMARY}

To assess the efficiency of different treatments in reducing the effect of Dothistroma septospora on the growth of young Pinus radiata, six treatments and a control were defined. The treatments included fertilization, the use of herbicide or fungicide and a combination of both at plantation. For comparison, at plantation, two growth thresholds were treated annually with fungicide being one of them additionally treated with herbicide.

The diameter growth proved to be more efficient than height to assess the efect of treatments. Fungicide applied after one years significantly reduce the disease intensity, but did not overcome the threshold growth. Herbicide at plantation and fungicide after one year, exceeded the control growth in $20 \%$.

\section{INTRODUCCION}

E1 daño causado por Dothistroma septospora (Dorog.) Morelet a Pinus radiata D. Don aparenta justificar la aplicación de técnicas de control, sin embargo debe resolverse el problema de cuál de ellas seleccionar y, más aún, la medida en que se debe aplicar. Si bien es cierto que existen antecedentes en la literatura internacional acerca de la eficacia de algunos tratamientos, esta debe ser confirmada para las condiciones medioambientales de nuestro país. Entonces se estima necesario esta- blecer un ensayo que considere tratamientos alternativos para controlar que el daño no se limite sólo a la aplicación de fungicida, de modo que se obtenga como resultado la selección de una medida de control eficaz.

Normalmente, cuando se desea controlar el daño causado por el hongo se piensa en la aplicación de fungicidas cúpricos, dado que su eficacia ha sido probada (Gibson, 1971; Gilmour y Noorderhaven, 1972; Kershaw et al., 1979; White, 1976; Van der Pas, 1981; Woollons y Hayward, 1984). Sin embargo, es dable pensar que mediante

* Parte del Proyecto "Modelo de crecimiento para plantaciones jóvenes de Pinus radiata sometidas a diferentes tratamientos para el control de Dothistroma septospora inscrito en la Dirección de Investigación y Convenios UACH y financiado por CONAF, mediante Convenio Prospección Nacional Forestal. 


\section{R. H. CONTRERAS}

medidas indirectas que vigoricen el desarrollo de los árboles se puede obtener un incremento de su resistencia al ataque del hongo. Tal puede ser el caso de la aplicación de herbicida, dado que reduce la competencia del árbol con malezas por agua y nutrientes (Hermosilla y Martino, 1982). Del mismo modo la fertilización tiene igual efecto sobre el vigor del árbol y de un modo específico la fertilización con azufre disminuye la suceptibilidad a la infección por Dothistroma (Turner y Lamber, 1978).

El presente trabajo pretende esclarecer el efecto de tratamientos directos e indirectos para reducir la pérdida de crecimiento causada por el hongo considerando medidas como la aplicación de fungicidas cúpricos, fertilización y aplicación de herbicida, comparando los parámetros volumétricos de los árboles en sus tres primeros años de plantación.

\section{METODO EXPERIMENTAL}

El ensayo se estableció en 1984 en dos rodales homogéneos de Pino Insigne de segunda rotación establecidos el mismo año a $18 \mathrm{~km}$ al norte de Valdivia. La densidad inicial de plantación es de 1.600 pl/há con un espaciamiento de 2,5 x $2,5 \mathrm{~m}$. El sitio del ensayo se encuentra bajo un clima templado lluvioso del tipo costero occidental y en suelos serie Correltue.

El estudio corresponde a un proyecto de investigación que tiene un horizonte de planificación mayor (Peredo et al., 1985) y que tiene un diseño de bloques completos al azar con parcelas permanentes de $400 \mathrm{~m}^{2}$ $(20$ x $20 \mathrm{~m})$ en un número de 51 . Se estudiaron seis tratamientos y un testigo con un número variable de repeticiones que va de tres a $24^{1}$.

- Aplicación de fungicida todos los años (Ft/a), con seis repeticiones.

- Testigo ( Te), con seis repeticiones.

- Aplicación de herbicida en el año cero y fungicida todos los años (HoFt/a), con tres repeticiones.

Esto se debe a que de los 17 tratamientos del proyecto señalado 10 aún no entraban en vigencia en 1986, siendo asimiladas sus parcelas a los vigentes para así aumentar su base de datos.
- Aplicación de herbicida y fungicida en el año cero (HoFo), con tres repeticiones.

- Aplicación de herbicida en el año cero (Ho), con tres repeticiones.

- Aplicación de fungicida en el año cero (Fo), con 24 repeticiones.

- Fertilización en el año uno $\left(\mathrm{Fe}_{1}\right)$, con seis repeticiones.

Los tratamientos Ft/a y HoFt/a son umbrales biológicos de crecimiento con bajos niveles de daño y el Te el que soporta la mayor pérdida de crecimiento con altos niveles de daño.

Las especificaciones de los tratamientos son:

- La aplicación de herbicida se practicó en septiembre de 1984, en una dosis de 3 kg/há del producto Velpar (MR Dupont) mediante Spot-Gun.

- La aplicación de fungicida, en noviembre, consiste en una mezcla de polvos mojables Cupravit Forte y Antracol, combinados en una proporción 65 y $35 \%$ de cada producto respectivamente, en una dosis de 2,5 y $5,0 \mathrm{~kg} / \mathrm{há}$ en 1984 y 1985 respectivamente.

- La fertilización consiste en $80 \mathrm{gr} / \mathrm{pl} \mathrm{de}$ Superfosfato triple, $8 \mathrm{gr} / \mathrm{pl}$ de Boronatrocalcita, $100 \mathrm{gr} / \mathrm{pl}$ de Salitre potásico y $50 \mathrm{gr} / \mathrm{pl} \mathrm{de} \mathrm{Sulfato}$ de magnesio.

Todos los años se midió la intensidad del daño como porcentaje de copa atacada en el mes de agosto y los parámetros volumétricos diámetro de cuello ( $\mathrm{D}, \mathrm{mm}$ ) y altura $(\mathrm{H}, \mathrm{cm})$. Estos últimos se tratan como diferenciales de crecimiento o incrementos $(\triangle \mathrm{D}$ y $\triangle \mathrm{H})$ desde 1984 descontando su valor inicial.

Los tratamientos se analizan mediante la prueba de comparación múltiple de Scheffe, luego de comprobarse la distribución normal de los datos, determinando el efecto de los tratamientos sobre la intensidad del daño y los parámetros volumétricos. Se aprovecha también el ordenamiento creciente de los tratamientos en base a los valores de la variable analizada que hace la prueba, orden que asigna el primer lugar para el tratamiento que alcanza el menor valor de la variable y el séptimo para el mayor. 
RESULTADOS

Comparación de los tratamientos sobre la intensidad del daño: En la primera medición de daño, realizada en 1984, las plantas no presentaron ataque, por lo que todas se iniciaron en el ensayo en igualdad de condiciones. Al año siguiente ya aparecen los síntomas de la enfermedad sin superar un $30 \%$ (Cuadro 1).

\section{CUADRO 1}

Comparación de los tratamientos mediante la intensidad del daño promedio (\% de copa atacada).

Comparison of treatments by mean damage intensity (\% of damaged crown).

\begin{tabular}{|c|c|c|c|c|c|c|c|}
\hline \multicolumn{8}{|c|}{ Año 1985} \\
\hline $\mathrm{T}$ & $\mathrm{Ft} / \mathrm{a}$ & Fo & HoFo & Ho & $\mathrm{Fe}$, & $\mathrm{Te}$ & $\mathrm{HoFt} / \mathrm{a}$ \\
\hline$\underline{\bar{x}}$ & 10,5 & 13,6 & 14,0 & 19,1 & 22,7 & 25,0 & 27,9 \\
\hline$\overline{\mathrm{Sx}}$ & 8,3 & 13,2 & 12,1 & 15,3 & 24,5 & 21,5 & 28,9 \\
\hline \multicolumn{8}{|c|}{ Año 1986} \\
\hline $\mathrm{T}$ & $\mathrm{Ft} / \mathrm{a}$ & $\mathrm{HoFt} / \mathrm{a}$ & HoFo & Ho & Fo & $\mathrm{Te}$ & $\mathrm{Fe}_{1}$ \\
\hline$\underline{\bar{X}}$ & 20,1 & 33,0 & 52,7 & 54,2 & 59,7 & 60,4 & 60,9 \\
\hline$\overline{\mathrm{Sx}}$ & 13,2 & 20,2 & 24,3 & 19,4 & 22,2 & 22,0 & 26,2 \\
\hline
\end{tabular}

El análisis estadístico entrega las siguientes diferencias significativas de intensidad de daño:

$\begin{array}{lll}\mathrm{Ho}, \mathrm{Fe}_{1}, & & \\ \text { Te y HoFt/a } & > & \\ \mathrm{Fe}_{1}, \mathrm{Te} & & \\ \text { y HoFt/a } & >\mathrm{HoFo} \\ \mathrm{HoFt} / \mathrm{a} & > & \mathrm{Ho}\end{array}$

Todos los tratamientos que incluyen fungicida se comportan del mismo modo, siendo los menos atacados, con excepción de HoFt/a que se comporta al margen de lo esperado, y los tratamientos $\mathrm{Fe}_{1}$ y $\mathrm{Te}$ se destacan como los más atacados.

En 1986 la diferencia entre tratamientos se acrecienta, donde la oscilación de los niveles de daño va de 10 a $61 \%$. El menos atacado Ft/a se destaca como significativamente distinto de todos los demás, lo mismo ocurre con HoFt/a, que está más atacado que Ft/a y menos que los restantes, recuperándose de la anormalidad ocurrida en 1985. Es importante destacar que todos los demás tratamientos no se diferencian del Te, manteniendo un alto daño.

\begin{tabular}{lccr} 
Comparación de los & \multicolumn{2}{c}{ tratamientos sobre } \\
parámetros volumétricos: & La pérdida de \\
crecimiento acumulada & para el período
\end{tabular}

1984-1986 por el testigo respecto del umbral Ft/a es de 8,3 y $23,7 \%$ en $H$ y D respectivamente (Cuadro 2). Los tratamientos que incluyen herbicida son, en promedio, un 3 y $9 \%$ más pequeños que $\mathrm{Ft} / \mathrm{a}$ en $\Delta \mathrm{H}$ y $\Delta \mathrm{D}$ respectivamente y desde otro punto de vista, los mismos superan en un 5,8 y $18,8 \%$ al testigo en $\Delta H \quad y \quad \Delta D$ respectivamente.

La comparación estadística de los tratamientos desglosada por parámetro volumétrico es:

Diferencial de altura: Al primer año del ensayo se detecta que HoFt/a es significativamente menor que Te, Ft/a y HoFo, dándose un hecho inesperado al ocupar HoFt/a el primer lugar del orden creciente (Cuadro 3). Por otra parte, ninguno de los tratamientos se diferencia del Te, lo que indica que no se detectan efectos de los mismos.

En 1986 se aclara el efecto de los tratamientos, diferenciándose entre sí, encontrándose las siguientes relaciones derivadas del cuadro 3 :

$\begin{array}{lll}\text { Ho, Ho Fo } & & \\ \text { y Ft/a } & & \mathrm{Fe}_{1} \\ \mathrm{Ho} \text { Fo y Ft/a } & > & \mathrm{Fo} \\ \mathrm{Ft} / \mathrm{a} & & >\mathrm{Te}\end{array}$




\section{CUADRO 2}

Crecimientos relativos diámetro y altura al cabo de dos años del ensayo.

Relative height and diameter growth after two years.

\begin{tabular}{cccccccc}
\hline $\begin{array}{l}\Delta H \\
\mathrm{~T}\end{array}$ & & $\mathrm{Fo}$ & $\mathrm{Te}$ & $\mathrm{HoFt} / \mathrm{a}$ & $\mathrm{Ho}$ & $\mathrm{HoFo}$ & $\mathrm{Ft} / \mathrm{a}$ \\
$*(\%)$ & 12,1 & 8,9 & 8,3 & 6,1 & 2,7 & 0,1 & \\
$* *(\%)$ & $-4,2$ & $-0,7$ & & 2,4 & 6,0 & 8,9 & 9,0 \\
$\Delta \mathrm{D}$ & & $\mathrm{Fo}$ & $\mathrm{Fe}_{\mathrm{t}}$ & $\mathrm{HoFo}$ & $\mathrm{Ho}$ & $\mathrm{HoFt} / \mathrm{a}$ & $\mathrm{Ft} / \mathrm{a}$ \\
$\mathrm{T}$ & $\mathrm{Te}$ & 22,4 & 15,8 & 12,8 & 8,6 & 6,6 & \\
$*(\%)$ & 23,7 & 1,7 & 10,3 & 14,2 & 19,8 & 22,4 & 31,0 \\
$*(\%)$ & & & & & & & \\
\hline
\end{tabular}

* Pérdida porcentual de crecimiento de los tratamientos respecto del umbral Ft/a (100\%).

** Mejora porcentual del crecimiento sobre el testigo (100\%).

\section{CUADRO 3}

Comparaciones múltiples de los tratamientos sobre la base de las diferenciales de crecimiento en altura y diámetro $(\triangle \mathrm{H}$ y $\Delta \mathrm{D})$.

Multiple comparison of treatments based on differential height and diameter growth.

Año 1985

Variable Comparación múltiple de tratamientos

$\begin{array}{cccccccc}\Delta \mathrm{H} & \mathrm{HoFt} / \mathrm{a} & \mathrm{Fe}_{\mathrm{i}} & \mathrm{Fo} & \mathrm{Ho} & \mathrm{Te} & \mathrm{Ft} / \mathrm{a} & \mathrm{HoFo} \\ \Delta \mathrm{D} & \mathrm{Fo} & \mathrm{Te} & \mathrm{Fe}_{1} & \mathrm{HoFt} / \mathrm{a} & \mathrm{Ft} / \mathrm{a} & \mathrm{HoFo} & \mathrm{Ho}\end{array}$

Año 1986

\begin{tabular}{|c|c|c|c|c|c|c|c|}
\hline$\Delta \mathrm{H}$ & $\mathrm{Fe}_{1}$ & Fo & $\mathrm{Te}$ & $\mathrm{HoFt} / \mathrm{a}$ & Ho & HoFo & $\mathrm{Ft} / \mathrm{a}$ \\
\hline$\Delta \mathrm{D}$ & $\mathrm{Te}$ & Fo & $\mathrm{Fe}_{1}$ & HoFo & Ho & $\mathrm{HoFt} / \mathrm{a}$ & $\mathrm{Ft} / \mathrm{a}$ \\
\hline
\end{tabular}

Es importante destacar que el umbral Ft/a es el único que supera al Te y que los restantes no tienen un crecimiento en altura distinto de este. Con todo, al analizar el orden creciente de los tratamientos se encuentra a HoFt/a en cuarta ubicación, cuando debiera ocupar la $6^{\mathrm{a}}-$ o $7^{\mathrm{a}}$-, que son las de mayor crecimiento, por tratarse de un umbral. Del mismo modo, $\mathrm{Fe}_{1}$ y Fo son menores al testigo aunque no significativamente.

Diferencial de diámetro de cuello: En 1985 la comparación múltiple de tratamientos indica que Fo y Te han incrementado su diámetro en forma significativamente menor que Ho (Cuadro 3). Al igual como ocurre para $\Delta H$ se dan resultados inesperados como que los umbrales $\mathrm{HoFt} / \mathrm{a}$ y $\mathrm{Ft} / \mathrm{a}$ se ubiquen en $4^{\mathrm{a}}-\mathrm{y} 5^{\mathrm{a}}-$ posición, siendo superados por HoFo y Ho, aunque no significativamente.

En 1986, tanto la diferenciación como el ordenamiento creciente de los tratamientos mejora acercándose más a lo esperado, de tal manera que Te y Fo tienen un crecimiento diamétrico significativamente menor que los demás tratamientos, igual 
cosa ocurre con $\mathrm{Fe}_{1}$ y $\mathrm{HoFo}$ al ser significativamente menores que Ft/a. Es importante destacar que $\mathrm{HoFt} / \mathrm{a}$ y $\mathrm{Ft} / \mathrm{a}$ se ubican en las posiciones de mayor crecimiento $\left(6^{a}-\right.$ y $\left.7^{\mathrm{a}}-\right)$, como era de esperar por ser umbrales, y Te ocupa la menor $\left(1^{\mathrm{a}}\right)$. También es destacable que Ho no es distinto de ambos umbrales.

\section{DISCUSION}

La comparación de tratamientos respecto de la intensidad del daño muestra en 1985 que tres de los cuatro tratamientos que fueron aplicados con fungicida en noviembre de 1984 presentan un daño significativamente menor que Te. El caso de $\mathrm{HoFt} / \mathrm{a}$ es singular al no resultar eficaz para controlar el daño, mostrándose como el más dañado, probablemente debido a alguna falla en la aplicación del mismo en 1984. Esto resulta deducible al constatar que en 1986 remonta posiciones hasta colocarse en una que indica un daño inmediatamente superior al otro umbral (Ft/a), debido probablemente a que en la aplicación de 1985 se superó la falla.

Los tratamientos Ho y $\mathrm{Fe}_{1}$ no son distintos del testigo en 1985, con todo, son capaces de reducir el daño aunque no a niveles significativos.

En 1986 sólo Ft/a y HoFt/a logran un daño significativamente menor que los demás, existiendo también diferencia entre ellos, ya que el primero presenta menos ataque que el segundo. Todos los restantes no difieren entre sí, incluso HoFo y Ho, lo que indica que aplicar fungicida el año de establecimiento no lleva a diferenciación con el testigo, resultando infructuoso dos años después de la aplicación. Este tratamiento protegió a las plantas en noviembre de 1984 reduciendo el daño hasta agosto del año siguiente a la mitad del presentado por el testigo. Como no se aplicó fungicida en 1985, al año siguiente se perdió toda protección registrando un daño similar al testigo.

Teniendo en cuenta el daño del testigo en 1985 y $1986,25,0$ y $60,4 \%$ respectivamente, puede calificarse al primer año como de un ataque moderado y al segundo como de alto ataque del hongo. En 1985 se logra alguna distinción entre los tratamientos que no son umbrales, dado un nivel de daño moderado. Cuando el daño es alto estos tratamientos no son capaces de reducir significativamente la intensidad del ataque. Es así como Ho reduce la intensidad del daño en 1985 aunque no significativamente, pero en 1986 no es capaz de hacerlo.

La comparación del crecimiento de los tratamientos respecto de Ft/a (Cuadro 2) indica que la altura es un parámetro menos sensible a la defoliación causada por Dothistroma, lo que se ve confirmado por Van der Pas (1981) y Kershaw et al., (1979).

Con la aplicación de herbicida se recupera parte del crecimiento perdido, reduciéndose la diferencia a menos de la mitad, tanto para $\Delta H$ como para $\Delta D$, como consecuencia de la combinación de liberación de competencia y menor daño, aunque no alcanzó diferencia significativa.

Al revisar la comparación estadística de los tratamientos se confirma la menor sensibilidad al daño de la altura, al no detectarse una clara diferenciación de crecimiento entre ellos en un año de crecimiento, siendo necesario acumular el efecto de un segundo año (1986) para que surja; con todo, en 1986 sólo el umbral Ft/a logra superar significativamente el crecimiento en altura del testigo.

Respecto del crecimiento diamétrico se tiene una respuesta más rápida a los tratamientos; ya en 1985 aparecen diferencias significativas y un ordenamiento creciente cercano a lo esperado. Sin embargo, al año siguiente mejora la claridad de los efectos por tratamiento y su orden creciente, por lo que el diámetro es el parámetro más apropiado para compararlos.

Los menores crecimientos los presentan el Te y Fo; siendo iguales se confirma que aplicar fungicida al establecimiento no lleva a mejorar significativamente el crecimiento en diámetro, como se señalara al comprobar que no tienen un nivel de daño distinto. El tratamiento $\mathrm{Fe}_{1}$ resultó ineficaz, al no ser distinto del Te y significativamente menor que Ft/a, debido a un defectuoso control de malezas practicado previo a la fertilización, perdiendo el ferti- 
lizante en la competencia con malezas (Gerding et al., 1986 y 1987).

Ante la decisión de controlar el daño, Ft/a y HoFt/a resultan ineficientes desde un punto de vista económico, por el costo que implican. Fo y $\mathrm{Fe}_{1}$ no son alternativas viables, aunque es necesario profundizar y mejorar la fertilización como medida de control. Restan entonces Ho y HoFo, el primero no tiene un crecimiento diamétrico significativamente menor que Ft/a, por lo que junto a su costo más bajo lo indican como una alternativa viable; HoFo no es significativamente menor al otro umbral HoFt/a, lo que también lo hace viable- sin embargo, como ya se probara la ineficacia de Fo, es recomendable hacer la aplicación de fungicida en el año uno $\left(\mathrm{HoFe}_{1}\right)$. El fundamento a esta sugerencia está en dejar a la planta sólo con las aplicaciones practicadas en vivero el primer año de establecida, y aplicar al cabo de este tiempo para aumentar la protección por otro año, lográndose al menos los dos primeros años de crecimiento con daño controlado para obtener plantas con un diámetro a lo menos un $19,8 \%$ mayor que aquellas no tratadas, valor que alcanzó Ho en 1986 (Cuadro 2).

A la luz de lo anterior, se constata la existencia de tratamientos alternativos para reducir la pérdida de crecimiento que no sólo consideran la aplicación de fungicida, de tal manera que combinándolo con herbicida, o sólo este último, se logra una recuperación de crecimiento importante, que no es del todo atribuible a una mayor resistencia producto de la liberación de competencia, dado que Ho no fue capaz de reducir significativamente la intensidad del daño.

\section{CONCLUSIONES}

- Al primer año de crecimiento una aplicación de fungicida de $2,5 \mathrm{~kg} / \mathrm{há}$ reduce significativamente la intensidad del daño causado por Dothistroma respecto del testigo.

- Al cabo de dos años de crecimiento sólo con la aplicación anual de fungicida se reduce significativamente el ataque en el segundo año.
- Desde el punto de vista del daño y del crecimiento Fo no es capaz de superar en crecimiento al testigo.

- La altura de la planta es menos afectada que el diámetro de cuello por el daño causado por el hongo.

- Es fundamental controlar malezas por a lo menos una temporada de crecimiento al momento de fertilizar.

- Los tratamientos Ho y HoFo son eficaces en la recuperación de la pérdida de crecimiento diamétrico del Te respecto de Ft/a.

- Se recomienda la aplicación de herbicida al establecimiento y fungicida un año más tarde para superar en al menos un $20 \%$ al testigo.

\section{REFERENCIAS}

GERDING, V.; SCHLATTER, J. y BARRIGA, L. 1986. "Fertilización para el establecimiento de Pinus radiata D. Don en Valdivia". Bosque 7 (2): 121-128

GERDING, V.; SCHLATTER, J. y GOMEZ, F. 1987. "Evaluación de cinco años de una fertilización al establecimiento en Pinus radiata D. Don, Valdivia", Acta V Simposio Nac. de la Ciencia del Suelo. Soc. Ch. de la Ciencia del Suelo. 99-105, Santiago.

GIBSON, I.A.S. 1971. "Field control of Dothistroma bligth of Pinus radiata using copper fungicide sprays". E. Afr. Agric. For. J. Kenia, 247-274.

Comp. 1979. Diseases of forest trees widely planted as exotics in the tropics and southern hemisphere. Kew, Surrey. University of Oxford. V. 2: 73-76.

GILMOUR, J.W. y NOORDERHAVEN, A. 1972 "Control of Dothistroma needle bligth by low volume aerial application of copper fungicides". N.Z.J. For. Sci. 3(1): 120-136.

HERMOSILLA, M. y MARTINO, I. 1982. "Influencia del uso de herbicida, diferentes calidades de plantas y fertilizante sobre la productividad de un sitio". En Evaluación de la productividad de sitios forestales. Reunión de trabajo. Universidad Austral de Chile, Valdivia. Actas pp. 245-255.

KERSHAW, D.J.; GADGIL, P.D.; LEGGAT, G.J.; RAY, J.W. y VAN DER PAS, J.B. 1979. "Handbook for the assessment and control of Dothistroma needle bligth". N.Z. Forest Service, 43 pp.

PAS, J.B. VAN DER. 1981. "Reduced early growth rates of Pinus radiata caused by Dothistroma pini", N.Z.J. For. Sci. 11(3): 210-220.

; BULMAN, L. y HORGAN, G.P. 1984. "Disease control by aerial spraying of Dothistroma pini in tended stands of Pinus radiata in New Zealand". N.Z.J. For. Sci. 13(1): 23-40.

PEREDO, H.L.; OLIVARES, B.; MENESES, M. y BELLO, F. 1984. Evaluación del daño y control químico de Dothistroma pini. Valdivia, Universidad Austral de Chile. Fac. Cien. For., Serie Tecn., Informe de Convenio 69, $22 \mathrm{pp}$.

PEREDO, H.; BELLO, F. y CONTRERAS, R. 1985. Modelo de crecimiento para plantaciones jóvenes 
de Pinus radiata, sometidas a diferentes tratamientos para el control de Dothistroma septospora. I Diseño experimental. Valdivia, Chile. Universidad Austral de Chile, Facultad de Ciencias Forestales. Serie Técnica, Informe de Convenio. 15 pp.

TURNER, J. y LAMBERT, M.J. 1978. "Sulphur nutrition of conifers in relation to response to fertilizer nitrogen, to fungal infections and to soil parent materials". In Forest Soils and Land Use. Proce- eding of the fifth North American Forest Soil Conference. Colorado State University, sp.

WHITE, A.G.D. 1976. "Spraying pine plantations with fungicides - the managers dilemma". Forest. Ecol. Manage 1: 7-19.

WOOLLONS, R.C. y HAYWARD, W.J. 1984. "Growth losses in Pinus radiata stands unsprayed for Dothistroma pini". N. Z. J. For. Sci. 14(1): 14-22. 